\title{
Reduced risk of dyslipidaemia with oolong tea consumption: a population-based study in southern China
}

\author{
Deqing $\mathrm{Yi}^{1,2} \dagger$, Xuerui $\operatorname{Tan}^{1} \dagger$, Zhiguo Zhao, ${ }^{1,2}$, Yingmu Cai ${ }^{1}$, Yiming $\mathrm{Li}^{1}$, Xiuying $\operatorname{Lin}^{3}$, Sailan $\mathrm{Lu}^{3}$, \\ Yongsong Chen ${ }^{1}$ and Qingying Zhang ${ }^{1,2 *}$ \\ ${ }^{1}$ The Work Group of Chronic Disease Surveillance, The First Affiliated Hospital of Shantou University Medical College, \\ Shantou, Guangdong 515041, People's Republic of China \\ ${ }^{2}$ Department of Preventive Medicine, Shantou University Medical College, Shantou, Guangdong 515041, \\ People's Republic of China \\ ${ }^{3}$ Health Care Center, The Chaonan Minsheng Hospital, Shantou, Guangdong 515144, People's Republic of China \\ (Submitted 1 May 2013 - Final revision received 24 September 2013 - Accepted 9 October 2013 - First published online 14 November 2013)
}

\section{Abstract}

Experimental studies have suggested that tea consumption could lower the risk of dyslipidaemia. However, epidemiological evidence is limited, especially in southern China, where oolong tea is the most widely consumed beverage. We conducted a population-based case-control study to evaluate the association between consumption of tea, especially oolong tea, and risk of dyslipidaemia in Shantou, southern China, from 2010 to 2011. Information on tea consumption, lifestyle characteristics and food consumption frequency of 1651 patients with newly diagnosed dyslipidaemia and 1390 controls was obtained using a semi-quantitative questionnaire. Anthropometric variables and serum biochemical indices were determined. Drinking more than $600 \mathrm{ml}$ (2 paos) of green, oolong or black tea daily was found to be associated with the lowest odds of dyslipidaemia risk $(P<0 \cdot 001)$ when compared with non-consumption, but only oolong tea consumption was found to be associated with low HDL-cholesterol levels. A dose-response relationship between duration of tea consumption and risk of dyslipidaemia (OR 0.10,95\% CI 0.06, 0.16), as well as that between amount of dried tea leaves brewed and risk of dyslipidaemia (OR 0.34, 95\% CI 0.24, 0.48), was found. Moreover, consumption of oolong tea for the longest duration was found to be associated with 3.22, 11.99 and 6.69\% lower blood total cholesterol, TAG and LDL-cholesterol levels, respectively. In conclusion, the present study indicates that long-term oolong tea consumption may be associated with a lower risk of dyslipidaemia in the population of Shantou in southern China.

Key words: Dyslipidaemia: Tea: Epidemiology: Risk: China

Dyslipidaemia, a major systemic disorder, is one of the key risk factors of CVD, and its morbidity is high and it is a leading contributor to mortality worldwide ${ }^{(1)}$ including China. The prevalence of dyslipidaemia is increasing in China ${ }^{(1,2)}$.

Tea, from the plant Camellia sinensis, is a widely consumed beverage in the world, especially in Shantou, southern China. People have a special way of preparing tea, called Gongfu tea. The teapot is as big as a fist and the teacups are as small as tiny liqueur glasses $(30 \mathrm{ml})$. Gongfu tea is prepared by brewing dried tea leaves with hot water. Drinking Gongfu tea is a pastime and a social activity to promote friendship and business negotiations in the region. These features might help us determine the relatively large variation in tea intake and measure tea intake with high reliability.

Tea contains many compounds, especially polyphenols, depending on the degree of fermentation. Tea can be classified into three major types: non-fermented green tea; partially fermented oolong tea; fully fermented black tea ${ }^{(3)}$. The composition of partially fermented oolong tea is somewhat similar to that of green tea. During the fermentation process, catechins are converted into the major black tea polyphenolic components theaflavins and thearubigins and other high-molecular weight components ${ }^{(4)}$.

Epidemiological studies have shown that polyphenolic compounds present in tea could reduce the risk of

\footnotetext{
Abbreviations: HDL-C, HDL-cholesterol; H-TAG, hypertriacylglycerolaemia; H-TC, hypercholesterolaemia; LDL-C, LDL-cholesterol; OTPP, oolong tea polymerised polyphenols; TC, total cholesterol.
}

*Corresponding author: Dr Q. Zhang, fax +86 75488259850 , email qyzhang@stu.edu.cn

† These authors contributed equally to the study. 
$\mathrm{CVD}^{(5-7)}$. Tea may protect against CVD by affecting lipid and lipoprotein levels. Animal studies have demonstrated blood levels of lipids to be lowered by the consumption of tea extracts $^{(4,8-10)}$, but results of clinical trials on the effect of tea consumption on serum lipid concentrations in human subjects have been inconsistent ${ }^{(11-15)}$. Although intervention studies had examined relatively large differences in tea intake among the groups compared, the duration of interventions was, in general, short and long-term tea consumption was not assessed ${ }^{(16-18)}$. The effect of tea consumption on dyslipidaemia requires a long duration to develop, so information on long-term tea exposure is important. To our knowledge, only a few epidemiological studies have shown an inverse relationship between green tea consumption and lipid levels ${ }^{(19-21)}$, but not for oolong tea consumption. Oolong tea contains oolong tea polymerised polyphenols (OTPP), derived from the process of semi-fermentation, which are not present in green tea. OTPP have stronger inhibitory effects on lipase than the total extracts of green tea in vitro ${ }^{(22)}$. Studies in mice have shown that oolong tea leaves have the strongest lowering effect on TAG levels ${ }^{(23)}$. The Shantou population mainly uses the semi-fermented oolong tea, so epidemiological evidence of an association with reduction of lipid levels is needed for this population.

The present population-based case-control study aimed to ascertain the association between consumption of tea, especially oolong tea, and risk of dyslipidaemia in Shantou, southern China.

\section{Methods}

\section{Study participants}

A population-based case-control study of dyslipidaemia was conducted in Shantou in southern China, from January 2010 to December 2010. The survey involved a questionnaire, medical examination, anthropometric parameter measurement and venous blood tests. All the participants aged 20-80 years had resided in Shantou for at least 5 years. The participants were randomly recruited from a cross-sectional survey of chronic diseases in Shantou, which was conducted in the health care centres of the First Affiliated Hospital of Shantou University Medical College and Chaonan Minsheng Hospital of Shantou. The required sample size was determined by assuming that $20 \%$ of southern Chinese adults drank 2 paos of tea daily with the relative risk of dyslipidaemia being 0.7 at such a level of intake, which was evaluated by our preliminary investigation. For a one-to-one case-control ratio at the $5 \%$ level of significance, the sample size needed to attain 90\% power was 1170 per group as calculated by NCSSPASS 2005. To further account for refusal and withdrawal, 1800 eligible cases and 1800 control subjects were approached and invited to participate in the study. A total of 1651 patients with dyslipidaemia and 1390 control subjects completed the entire interview, representing an eventual response rate of 91.7 and $77.2 \%$, respectively. Cases were diagnosed by the recommendations of the National Cholesterol Education Program $^{(24)}$. Selection criteria for cases were newly diagnosed dyslipidaemia with no history of dyslipidaemia and those for eligible controls were no history or clinical evidence of dyslipidaemia. Exclusion criteria for both cases and controls were major health problems such as heart diseases, stroke and cancer and use of medications that could interfere with lipid metabolism. The present study was conducted according to the guidelines laid down in the Declaration of Helsinki, and all procedures involving human subjects were approved by the Ethics Committee of the First Affiliated Hospital of Shantou University Medical College and Chaonan Minsheng Hospital. Written informed consent was obtained from all the subjects.

\section{Anthropometric parameter measurement}

Data were collected in examination centres at the hospitals. Body weight, height, waist circumference and blood pressure were measured by trained observers according to a standard protocol. Body weight and height were measured to the nearest $0 \cdot 1 \mathrm{~kg}$ and $0.1 \mathrm{~cm}$, respectively, with standardised equipment, and BMI was calculated as weight $(\mathrm{kg}) /$ height squared (m). Systolic blood pressure and diastolic blood pressure were averaged from three measurements.

\section{Questionnaire and exposure measurements}

A double-blind method was used for the questionnaire survey. Both the researchers and participants were blind to the dyslipidaemia status of the participants. Trained research staff used a standard questionnaire to collect demographic information and medical history. Demographic and lifestyle data included those on age, sex, education level (primary school, middle school, college or above), occupation (manual worker, office worker or retired/unemployed), smoking status (non-smoker or former/current smoker) and pack-years, alcohol drinking status (non-drinker or former/ current drinker), physical activity (frequency/d per week per month) and sitting time per $\mathrm{d}(<6,6-9$ or $>9 \mathrm{~h})$.

Information on the intake of common foods was obtained using a 125-item semi-quantitative FFQ developed for the southern Chinese population ${ }^{(25,26)}$. This validated instrument covered most of the food items commonly consumed in southern China. Intake frequency (per $\mathrm{d} /$ week/month) was recorded in detail. The reference recall period for dietary variables was set at 1 year before the interview process. In addition, we asked specific questions about tea consumption that had established reliability and reproducibility; intra-class correlation coefficients were $0 \cdot 82-0 \cdot 95^{(27)}$. The subjects were classified as 'ever' or 'never' tea drinkers (less than once per month). The ever-drinkers were asked to report their frequency of habitual intake in paos (about $300 \mathrm{ml}$, ten teacups of Gongfu tea: 1-3/month; 1-6/week; 1-2/d; 2-4/d; $>4 / \mathrm{d}$ ), duration of regular tea consumption (in years), types of tea consumed (green, black and oolong) and average quantity of dried tea leaves used for brewing (in grams) per year. For those who drank more than one type of tea, the reported ratio of consumption was used to estimate the respective frequency and amount of dried tea leaves for each type. 


\section{Laboratory methods and definition of dyslipidaemia}

All the examinations were carried out in the clinical laboratory at the hospitals. The concentrations of blood plasma total cholesterol (TC), TAG, HDL-cholesterol (HDL-C) and LDL-cholesterol (LDL-C), fasting plasma glucose, alanine aminotransferase, aspartate aminotransferase, uric acid and creatinine were measured by an enzymatic method using a Beckman autoanalyser (Beckman DXC800, Beckman Coulter, Inc.). Reagents of the same batch were used. Dyslipidaemia was defined as hypercholesterolaemia (H-TC; TC levels $\geq 5.18 \mathrm{mmol} / 1 \quad(2000 \mathrm{mg} / \mathrm{l})), \quad$ hypertriacylglycerolaemia (H-TAG; TAG levels $\geq 1.70 \mathrm{mmol} / \mathrm{l}(1500 \mathrm{mg} / \mathrm{l})$ ) or lower HDL-C (HDL-C levels $<1.04 \mathrm{mmol} / \mathrm{l}(400 \mathrm{mg} / \mathrm{l})$ ). These cutoffs were selected from the National Cholesterol Education Program recommendations ${ }^{(24)}$.

\section{Statistical analysis}

Descriptive statistics were used to summarise the characteristics of the participants and their tea consumption patterns. We examined the effects of tea consumption on dyslipidaemia risk using a logistic regression analysis of frequency of intake, duration of tea consumption and quantity of tea leaves brewed. We converted pao into $\mathrm{ml}$ for the analysis; 1 pao equates to ten teacups of Gongfu tea, about $300 \mathrm{ml}$. The frequency of tea drinking was classified into four levels $(<300 \mathrm{ml} /$ week, $\quad 300-1800 \mathrm{ml} /$ week, $\quad 300-600 \mathrm{ml} / \mathrm{d}$ and $>600 \mathrm{ml} / \mathrm{d}$ ), with the lowest level of intake being used as the reference category. For total and type-specific tea leaves used, we used the 33rd and 66th percentiles of these continuous variables among drinkers as cut-offs, which resulted in three increasing levels of exposure, with $<1 \mathrm{~g}$ /year as the reference category. To further assess the dose-response relationship between long-term tea exposure and dyslipidaemia risk, we used a linear trend analysis of duration of tea consumption and total and type-specific tea leaves brewed. Both crude and adjusted OR and 95\% CI were calculated. Finally, we used multiple linear regression to estimate the association between duration of oolong tea consumption and levels of blood lipids. The variables estimated from the linear regression model were used to calculate the absolute difference from the reference group. Percentage difference was calculated using the absolute reduction divided by the adjusted mean of the reference group. Adjusted mean lipid levels were calculated using least-squares means in the PROC GLM procedure of SAS for each category of duration of oolong tea consumption.

Table 1. Demographic characteristics of cases and controls in the present study carried out in Shantou, China (Mean values and standard deviations; percentages, odds ratios and $95 \%$ confidence intervals)

\begin{tabular}{|c|c|c|c|c|c|c|c|}
\hline & \multicolumn{2}{|c|}{ Cases (n 1651) } & \multicolumn{2}{|c|}{ Controls ( $n$ 1390) } & \multirow[b]{2}{*}{ OR } & \multirow[b]{2}{*}{$95 \% \mathrm{Cl}$} & \multirow[b]{2}{*}{$P$} \\
\hline & Mean & SD & Mean & SD & & & \\
\hline Age (years) & $43 \cdot 68$ & 11.46 & 38.57 & 11.67 & 1.04 & $1.03,1.05$ & $<0.001$ \\
\hline Sex, male (\%) & \multicolumn{2}{|c|}{82.9} & \multicolumn{2}{|c|}{66.4} & 0.41 & $0.35,0.50$ & $<0.001$ \\
\hline Education (\%) & & & & & & & 0.001 \\
\hline Primary school & \multicolumn{2}{|c|}{$16 \cdot 0$} & \multicolumn{2}{|c|}{$17 \cdot 4$} & 1.0 & & \\
\hline Middle school & \multicolumn{2}{|c|}{$22 \cdot 3$} & \multicolumn{2}{|c|}{27.4} & 1.12 & $0.90,1.41$ & \\
\hline College & \multicolumn{2}{|c|}{$61 \cdot 7$} & \multicolumn{2}{|c|}{$55 \cdot 2$} & 0.82 & $0.67,0.99$ & \\
\hline Occupation (\%) & & & & & & & 0.034 \\
\hline Manual worker & \multicolumn{2}{|c|}{24.1} & \multicolumn{2}{|c|}{26.5} & 1.0 & & \\
\hline Office worker & \multicolumn{2}{|c|}{$61 \cdot 2$} & \multicolumn{2}{|c|}{$61 \cdot 8$} & 1.09 & $0.92,1.29$ & \\
\hline Retired/unemployed & \multicolumn{2}{|c|}{14.7} & \multicolumn{2}{|c|}{11.7} & 1.38 & $1.09,1.76$ & \\
\hline BMI $\left(\mathrm{kg} / \mathrm{m}^{2}\right)$ & 24.90 & 3.15 & $22 \cdot 70$ & $3 \cdot 24$ & 1.24 & $1.21,1.28$ & $<0.001$ \\
\hline Waist circumference $(\mathrm{cm})$ & 84.57 & 8.91 & $77 \cdot 13$ & 10.03 & 1.09 & $1.08,1.10$ & $<0.001$ \\
\hline AST $(\mathrm{mmol} / \mathrm{l})$ & $28 \cdot 34$ & 10.75 & $25 \cdot 57$ & 9.19 & 1.03 & $1.02,1.04$ & $<0.001$ \\
\hline ALT $(\mathrm{mmol} / \mathrm{l})$ & 36.56 & 24.46 & $25 \cdot 89$ & 18.47 & 1.03 & $1.02,1.03$ & $<0.001$ \\
\hline $\mathrm{Cr}(\mathrm{mmol} / \mathrm{l})$ & $86 \cdot 10$ & 18.00 & $81 \cdot 81$ & 17.69 & 1.01 & $1.01,1.02$ & $<0.001$ \\
\hline $\mathrm{UA}(\mathrm{mmol} / \mathrm{l})$ & 411.05 & 81.08 & 369.57 & $75 \cdot 36$ & 1.01 & $1.00,1.01$ & $<0.001$ \\
\hline $\mathrm{SBP}(\mathrm{mmHq})$ & 123.48 & $17 \cdot 61$ & 116.92 & 14.65 & 1.03 & $1.02,1.03$ & $<0.001$ \\
\hline $\mathrm{DBP}(\mathrm{mmHg})$ & $80 \cdot 76$ & 11.46 & 76.00 & $10 \cdot 15$ & 1.04 & $1.04,1.05$ & $<0.001$ \\
\hline FPG $(\mathrm{mmol} / \mathrm{l})$ & \multirow{2}{*}{\multicolumn{2}{|c|}{$\begin{array}{r}5.34 \\
58.1\end{array}$}} & 5.00 & 0.92 & 1.42 & $1.30,1.56$ & $<0.001$ \\
\hline Smoking, yes (\%) & & & \multicolumn{2}{|c|}{42.1} & 1.91 & $1 \cdot 65,2 \cdot 20$ & $<0.001$ \\
\hline Smoking, pack-years & 14.64 & 18.89 & $8 \cdot 18$ & $14 \cdot 73$ & 1.03 & $1.02,1.03$ & $<0.001$ \\
\hline Alcohol drinking yes (\%) & \multirow{2}{*}{\multicolumn{2}{|c|}{46.4}} & \multicolumn{2}{|c|}{38.7} & 1.30 & $1.17,1.43$ & $<0.001$ \\
\hline Physical activity (times/week, \%) & & & & & & & 0.002 \\
\hline$<1$ & \multicolumn{2}{|c|}{69.7} & \multicolumn{2}{|c|}{64.0} & 1.0 & & \\
\hline 1 & \multicolumn{2}{|c|}{$10 \cdot 6$} & \multicolumn{2}{|c|}{$10 \cdot 6$} & 0.92 & $0.73,1.17$ & \\
\hline $2-3$ & \multicolumn{2}{|c|}{$11 \cdot 3$} & \multicolumn{2}{|c|}{$15 \cdot 0$} & 0.70 & $0.56,0.86$ & \\
\hline$>4$ & & & & & 0.74 & $0.57,0.94$ & \\
\hline Sitting time $(\mathrm{h} / \mathrm{d}, \%)$ & & & & & & & $<0.001$ \\
\hline$<6$ & & & & & 1.0 & & \\
\hline $6-9$ & & & & & 1.37 & $1.14,1.65$ & \\
\hline$>9$ & & & & & 2.50 & $2.02,3.06$ & \\
\hline
\end{tabular}

AST, aspartate aminotransferase; ALT, alanine aminotransferase; $\mathrm{Cr}$, creatinine; UA, uric acid; SBP, systolic blood pressure; DBP, diastolic blood pressure; FPG, fasting plasma glucose. 
Besides tea consumption, independent variables included in the regression models were age, sex, education level, BMI, physical activity, smoking status and pack-years, alcohol drinking status, and presence of hypertension and diabetes, together with weekly dietary intake of meat, fish, poultry, fruit and vegetables, with all being considered potential confounders from univariate analysis. A two-sided $P<0.05$ was considered statistically significant. All the data were double entered using Epi Info version 6.0 (Centers for Disease Control and Prevention) and then exported to SAS version 9.10 (SAS Institute, Inc.) for analysis. Power calculations involved the use of NCSS-PASS 2005.

\section{Results}

\section{Demographic characteristics}

Table 1 summarises the demographic characteristics of the participants. The study included 3041 subjects: 1651 with dyslipidaemia and 1390 controls. Among the cases were 1043 individuals with H-TC, 893 with H-TAG and 445 with L-HDL. According to univariate logistic regression, increased age, sex, education level and occupation were associated with dyslipidaemia (all $P<0 \cdot 05$ ). Patients with dyslipidaemia had higher BMI, waist circumference and blood pressure and

Table 2. Association of dietary components with dyslipidaemia among cases and controls* (Odds ratios and $95 \%$ confidence intervals)

\begin{tabular}{|c|c|c|c|c|c|c|c|}
\hline & \multicolumn{2}{|c|}{$\begin{array}{l}\text { Cases } \\
(n 1651)\end{array}$} & \multicolumn{2}{|c|}{$\begin{array}{l}\text { Controls } \\
(n 1390)\end{array}$} & \multirow[b]{2}{*}{ OR } & \multirow[b]{2}{*}{$95 \% \mathrm{Cl}$} & \multirow[b]{2}{*}{$P$} \\
\hline & $n$ & $\%$ & $n$ & $\%$ & & & \\
\hline Vegetables & & & & & & & 0.003 \\
\hline$<1$ time/week & 55 & $3 \cdot 3$ & 26 & 1.9 & $1 \cdot 0$ & & \\
\hline $1-6$ times/week & 47 & $2 \cdot 8$ & 63 & 4.5 & 0.35 & $0.19,0.64$ & \\
\hline$\geq 1$ time/d & 1549 & $93 \cdot 8$ & 1301 & $93 \cdot 6$ & 0.56 & $0.35,0.90$ & \\
\hline Fruit & & & & & & & $<0.001$ \\
\hline$<1$ time/week & 577 & 34.9 & 372 & $26 \cdot 8$ & 1.0 & & \\
\hline 1-6 times/week & 626 & 37.9 & 558 & $40 \cdot 1$ & 0.72 & $0.61,0.86$ & \\
\hline$\geq 1$ time/d & 448 & $27 \cdot 1$ & 460 & $33 \cdot 1$ & 0.63 & $0.52,0.75$ & \\
\hline Soya food & & & & & & & $<0.001$ \\
\hline$<1$ time/week & 1257 & $76 \cdot 1$ & 948 & $68 \cdot 2$ & $1 \cdot 0$ & & \\
\hline $1-6$ times/week & 299 & $18 \cdot 1$ & 306 & $22 \cdot 0$ & 0.74 & $0.62,0.88$ & \\
\hline$\geq 1$ time/d & 95 & $5 \cdot 8$ & 136 & $9 \cdot 8$ & 0.53 & $0.40,0.69$ & \\
\hline Milk & & & & & & & $<0.001$ \\
\hline$<1$ time/week & 1335 & 80.9 & 1019 & $73 \cdot 3$ & 1.0 & & \\
\hline $1-6$ times/week & 198 & $12 \cdot 0$ & 229 & $16 \cdot 5$ & 0.66 & $0.54,0.81$ & \\
\hline$\geq 1$ time/d & 118 & $7 \cdot 1$ & 142 & $10 \cdot 2$ & 0.63 & $0.49,0.82$ & \\
\hline Meat & & & & & & & 0.786 \\
\hline$<1$ time/week & 547 & $33 \cdot 1$ & 472 & $34 \cdot 0$ & $1 \cdot 0$ & & \\
\hline $1-6$ times/week & 734 & 44.5 & 620 & $44 \cdot 6$ & 1.02 & $0.87,1.20$ & \\
\hline$>1$ time $/ \mathrm{d}$ & 370 & $22 \cdot 4$ & 298 & 21.4 & 1.08 & $0.88,1.30$ & \\
\hline Animal offal & & & & & & & 0.001 \\
\hline$<1$ time/week & 864 & $52 \cdot 3$ & 803 & $57 \cdot 8$ & 1.0 & & \\
\hline $1-6$ times/week & 686 & 41.6 & 535 & 38.5 & $1 \cdot 20$ & $1 \cdot 03,1 \cdot 38$ & \\
\hline$\geq 1$ time/d & 101 & $6 \cdot 1$ & 52 & $3 \cdot 7$ & 1.80 & $1 \cdot 28,2 \cdot 56$ & \\
\hline Poultry & & & & & & & 0.658 \\
\hline$<1$ time/week & 344 & $20 \cdot 8$ & 275 & $19 \cdot 8$ & $1 \cdot 0$ & & \\
\hline $1-6$ times/week & 1006 & $60 \cdot 9$ & 869 & $62 \cdot 5$ & 0.93 & $0.77,1.11$ & \\
\hline$\geq 1$ time/d & 301 & $18 \cdot 2$ & 246 & $17 \cdot 7$ & 0.98 & $0.78,1.23$ & \\
\hline Egg & & & & & & & 0.529 \\
\hline$<1$ time/week & 292 & $17 \cdot 7$ & 236 & $17 \cdot 0$ & 1.0 & & \\
\hline $1-6$ times/week & 775 & $46 \cdot 9$ & 681 & $49 \cdot 0$ & 0.92 & $0.75,1.12$ & \\
\hline$\geq 1$ time/d & 584 & 35.4 & 473 & $34 \cdot 0$ & 0.99 & $0.81,1.23$ & \\
\hline Water fish & & & & & & & 0.917 \\
\hline$<1$ time/week & 427 & $25 \cdot 9$ & 355 & $25 \cdot 5$ & $1 \cdot 0$ & & \\
\hline 1-6 times/week & 491 & $29 \cdot 7$ & 423 & $30 \cdot 4$ & 0.97 & $0.80,1.17$ & \\
\hline$\geq 1$ time/d & 733 & 44.4 & 612 & $44 \cdot 0$ & 0.99 & $0.83,1.19$ & \\
\hline Seafood & & & & & & & 0.564 \\
\hline$<1$ time/week & 76 & $4 \cdot 6$ & 74 & $5 \cdot 3$ & 1.0 & & \\
\hline $1-6$ times/week & 1173 & $71 \cdot 0$ & 992 & 71.4 & $1 \cdot 15$ & $0.83,1.60$ & \\
\hline$\geq 1$ time/d & 402 & 24.4 & 324 & $23 \cdot 3$ & $1 \cdot 21$ & $0.85,1.72$ & \\
\hline Lard & & & & & & & $<0.001$ \\
\hline$<1$ time/week & 1036 & $62 \cdot 8$ & 949 & $68 \cdot 3$ & $1 \cdot 0$ & & \\
\hline 1-6 times/week & 245 & $14 \cdot 8$ & 214 & $15 \cdot 4$ & 1.05 & $0.86,1.29$ & \\
\hline$\geq 1$ time/d & 370 & $22 \cdot 4$ & 227 & $16 \cdot 3$ & 1.50 & $1 \cdot 24,1 \cdot 80$ & \\
\hline Pickle & & & & & & & $<0.001$ \\
\hline$<1$ time/week & 844 & $51 \cdot 1$ & 821 & $59 \cdot 1$ & $1 \cdot 0$ & & \\
\hline $1-6$ times/week & 480 & $29 \cdot 1$ & 372 & $26 \cdot 8$ & $1 \cdot 26$ & $1.06,1.48$ & \\
\hline$\geq 1$ time $/ \mathrm{d}$ & 327 & $19 \cdot 8$ & 197 & $14 \cdot 2$ & 1.62 & $1.32,1.98$ & \\
\hline
\end{tabular}

${ }^{*}$ OR and $P$ values refer to those of the controls. 
higher levels of fasting plasma glucose, alanine aminotransferase, aspartate aminotransferase, creatinine and urine acid than controls $(P<0.001)$ and increased cumulative smoking exposure, alcohol drinking status and sitting time, as well as reduced physical activity $(P<0 \cdot 01)$.

\section{Dietary characteristics}

The consumption of vegetables, fruit, soya food and milk at least once a week was strongly associated with a reduced risk of dyslipidaemia when compared with infrequent consumption or non-consumption $(P<0 \cdot 01$; Table 2$)$. In addition, the consumption of animal offal, lard and pickle at least once a week was strongly associated with an increased risk of dyslipidaemia when compared with infrequent consumption or non-consumption $(P<0 \cdot 01)$.

\section{Tea consumption patterns}

Table 3 summarises the results of univariate and multivariate logistic regression analyses of the association between frequency of tea consumption and risk of dyslipidaemia. The probability values were obtained using a likelihood ratio test of the overall significance of each variable of tea consumption in multivariate models. On carrying out a binary multivariate logistic regression analysis, with all the variables found to be significantly associated with dyslipidaemia risk in univariate analysis, drinking at least $300 \mathrm{ml}$ (1 pao) of tea per week was found to be associated with a decreased risk of dyslipidaemia when compared with infrequent consumption or

(Odds ratios and $95 \%$ confidence intervals) non-consumption $(P<0 \cdot 001)$, with the reduction in risk being most effective when drinking more than $600 \mathrm{ml}$ ( 2 paos) daily (adjusted OR 0.28, 95\% CI 0.20, 0.39). Similar results were obtained for the type of tea consumed $(P<0 \cdot 05)$.

The relationship between consumption of the three types of tea and components of dyslipidaemia was further analysed by multivariate logistic regression (Table 4). Consumption of all the three types of tea was associated with a reduced risk of H-TC, H-LDL and H-TAG, especially consumption of green tea (adjusted OR 0.26, 95\% CI 0.13, 0.53; adjusted OR 0.24, $95 \%$ CI $0.11,0.54$; adjusted OR 0.22, $95 \%$ CI $0.10,0.53$, respectively; $P<0 \cdot 001$ ), but only oolong tea, was significantly associated with L-HDL (adjusted OR 0.38, 95\% CI 0.26, 0.56; $P<0 \cdot 001$ ).

The effect of cumulative tea exposure and quantity of dried tea leaves brewed on dyslipidaemia is summarised Table 5 . Long duration of tea consumption, especially for more than 20 years, was associated with a reduced risk of dyslipidaemia (adjusted OR $0 \cdot 10,95 \% \mathrm{CI} 0 \cdot 06,0 \cdot 16, P<0 \cdot 001)$. In terms of the amount of tea leaves used, the dose-response relationship was significant overall $(P<0 \cdot 001)$. In particular, mean monthly consumption of green tea leaves of more than $750 \mathrm{~g}$ was associated with the largest reduction in dyslipidaemia risk (adjusted OR 0.37, 95\% CI 0.25, 0.54, P<0.001).

Furthermore, we analysed the association between cumulative oolong tea exposure and blood lipid levels using multiple linear regression models, with adjustment for variables significantly associated with dyslipidaemia (Table 6). The duration of tea consumption was linearly associated with blood TC, TAG and LDL-C levels $(P<0 \cdot 05)$. Compared with the reference

Table 3. Association between consumption of tea and risk of dyslipidaemia*

\begin{tabular}{|c|c|c|c|c|c|c|c|c|}
\hline & \multicolumn{2}{|c|}{$\begin{array}{l}\text { Cases } \\
(n 1651)\end{array}$} & \multicolumn{2}{|c|}{$\begin{array}{l}\text { Controls } \\
(n 1390)\end{array}$} & \multirow[b]{2}{*}{ Crude OR } & \multirow[b]{2}{*}{$95 \% \mathrm{Cl}$} & \multirow[b]{2}{*}{ Adjusted OR† } & \multirow[b]{2}{*}{$95 \% \mathrm{Cl}$} \\
\hline & $n$ & $\%$ & $n$ & $\%$ & & & & \\
\hline Total tea & & & & & & & $P<0.001$ & \\
\hline$<300 \mathrm{ml} \neq /$ week§ & 155 & 9.4 & 82 & 5.9 & 1.0 & & 1.0 & \\
\hline $300-1800 \mathrm{ml} /$ week & 98 & 5.9 & 113 & $8 \cdot 1$ & 0.46 & $0.31,0.68$ & 0.31 & $0.20,0.49$ \\
\hline $300-600 \mathrm{ml} / \mathrm{d}$ & 467 & $28 \cdot 3$ & 438 & 31.5 & 0.57 & $0.42,0.76$ & 0.31 & $0.22,0.44$ \\
\hline$>600 \mathrm{ml} / \mathrm{d}$ & 931 & $56 \cdot 4$ & 757 & 54.5 & 0.65 & $0.49,0.86$ & 0.28 & $0.20,0.39$ \\
\hline Green tea & & & & & & & $P<0.001$ & \\
\hline$<300 \mathrm{ml} \ddagger$ week§ & 1480 & $89 \cdot 6$ & 1178 & 84.7 & 1.0 & & 1.0 & \\
\hline $300-1800 \mathrm{ml} /$ week & 63 & $3 \cdot 8$ & 68 & 4.9 & 0.74 & $0.52,1.05$ & 0.79 & $0.53,1.19$ \\
\hline $300-600 \mathrm{ml} / \mathrm{d}$ & 79 & $4 \cdot 8$ & 106 & $7 \cdot 6$ & 0.59 & $0.44,0.80$ & 0.47 & $0.34,0.66$ \\
\hline$>600 \mathrm{ml} / \mathrm{d}$ & 29 & $1 \cdot 8$ & 38 & $2 \cdot 7$ & 0.61 & $0.37,0.99$ & 0.44 & $0.26,0.77$ \\
\hline Oolong tea & & & & & & & $P<0.001$ & \\
\hline$<300 \mathrm{ml} \neq /$ week§ & 229 & 13.9 & 161 & 11.6 & 1.0 & & 1.0 & \\
\hline $300-1800 \mathrm{ml} /$ week & 93 & $5 \cdot 6$ & 116 & $8 \cdot 4$ & 0.56 & $0.40,0.79$ & 0.47 & $0.32,0.69$ \\
\hline $300-600 \mathrm{ml} / \mathrm{d}$ & 492 & $29 \cdot 8$ & 441 & $31 \cdot 7$ & 0.78 & $0.62,0.99$ & 0.53 & $0.40,0.70$ \\
\hline$>600 \mathrm{ml} / \mathrm{d}$ & 837 & 50.7 & 672 & $48 \cdot 3$ & 0.88 & $0.70,1 \cdot 10$ & 0.46 & $0.35,0.60$ \\
\hline Black tea & & & & & & & $P=0.020$ & \\
\hline$<300 \mathrm{ml}$ /week§ & 1495 & $90 \cdot 6$ & 1219 & $87 \cdot 7$ & 1.0 & & 1.0 & \\
\hline $300-1800 \mathrm{ml} /$ week & 34 & $2 \cdot 1$ & 38 & $2 \cdot 7$ & 0.73 & $0.46,1.17$ & 0.88 & $0.51,1.51$ \\
\hline $300-600 \mathrm{ml} / \mathrm{d}$ & 85 & $5 \cdot 1$ & 79 & $5 \cdot 7$ & 0.88 & $0.64,1.20$ & 0.80 & $0.56,1.14$ \\
\hline$>600 \mathrm{ml} / \mathrm{d}$ & 37 & $2 \cdot 2$ & 54 & 3.9 & 0.56 & $0.37,0.86$ & 0.50 & $0.31,0.80$ \\
\hline
\end{tabular}

* The crude OR, adjusted OR and $P$ values refer to those of the controls.

†Adjusted for age; sex; education level; BMI; waist circumference; systolic blood pressure; diastolic blood pressure; levels of fasting plasma glucose, alanine aminotransferase, aspartate aminotransferase, creatinine and uric acid; physical activity; sitting time; smoking status; smoking pack-years; alcohol drinking status and weekly dietary intake of vegetables, fruit, soya food, milk, animal offal, lard and pickle.

$\ddagger 300 \mathrm{ml}$ equate to 1 pao.

$\S$ Referent. 
Table 4. Association between frequency of tea type consumed and components of dyslipidaemia (Odds ratios and $95 \%$ confidence intervals)

\begin{tabular}{|c|c|c|c|c|c|c|c|c|}
\hline & \multicolumn{8}{|c|}{ Components of dyslipidaemia } \\
\hline & \multicolumn{2}{|c|}{$\mathrm{H}-\mathrm{TC}(n$ 1043) } & \multicolumn{2}{|c|}{ H-TAG ( $n$ 893) } & \multicolumn{2}{|c|}{ H-LDL-C ( $n$ 540) } & \multicolumn{2}{|c|}{ L-HDL-C ( $n$ 445) } \\
\hline & $\mathrm{OR}^{*}$ & $95 \% \mathrm{Cl}$ & $\mathrm{OR}^{\star}$ & $95 \% \mathrm{Cl}$ & $\mathrm{OR}^{*}$ & $95 \% \mathrm{Cl}$ & $\mathrm{OR}^{*}$ & $95 \% \mathrm{Cl}$ \\
\hline Green tea & \multicolumn{2}{|c|}{$P<0.001$} & \multicolumn{2}{|c|}{$P<0.001$} & \multicolumn{2}{|c|}{$P<0.001$} & \multicolumn{2}{|c|}{$P=0.509$} \\
\hline$<300 \mathrm{ml} \dagger /$ week $\ddagger$ & $1 \cdot 0$ & & $1 \cdot 0$ & & $1 \cdot 0$ & & 1.0 & \\
\hline $300-1800 \mathrm{ml} /$ week & 0.51 & $0.31,0.85$ & 0.92 & $0.56,1.49$ & 0.56 & $0.30,1.05$ & 1.37 & $0.78,2.41$ \\
\hline $300-600 \mathrm{ml} / \mathrm{d}$ & 0.42 & $0.28,0.62$ & 0.43 & $0.27,0.68$ & 0.41 & $0.25,0.68$ & 0.95 & $0.59,1.53$ \\
\hline$>600 \mathrm{ml} / \mathrm{d}$ & 0.26 & $0.13,0.53$ & 0.24 & $0.11,0.54$ & 0.22 & $0.10,0.53$ & 0.65 & $0.31,1.37$ \\
\hline Oolong tea & \multicolumn{2}{|c|}{$P<0.001$} & \multicolumn{2}{|c|}{$P<0.001$} & \multicolumn{2}{|c|}{$P<0.001$} & \multicolumn{2}{|c|}{$P<0.001$} \\
\hline$<300 \mathrm{ml} \dagger /$ week $\ddagger$ & $1 \cdot 0$ & & $1 \cdot 0$ & & $1 \cdot 0$ & & 1.0 & \\
\hline $300-1800 \mathrm{ml} /$ week & 0.43 & $0.27,0.67$ & 0.51 & $0.31,0.85$ & 0.48 & $0.27,0.85$ & 0.38 & $0.20,0.67$ \\
\hline $300-600 \mathrm{ml} / \mathrm{d}$ & 0.51 & $0.37,0.71$ & 0.58 & $0.40,0.83$ & 0.45 & $0.30,0.67$ & 0.46 & $0.31,0.70$ \\
\hline$>600 \mathrm{ml} / \mathrm{d}$ & 0.45 & $0.33,0.62$ & 0.49 & $0.35,0.70$ & 0.46 & $0.32,0.67$ & 0.37 & $0.26,0.56$ \\
\hline Black tea & \multicolumn{2}{|c|}{$P<0.001$} & \multicolumn{2}{|c|}{$P=0.004$} & \multicolumn{2}{|c|}{$P=0.020$} & \multicolumn{2}{|c|}{$P=0.528$} \\
\hline$<300 \mathrm{ml} † /$ week $\ddagger$ & $1 \cdot 0$ & & $1 \cdot 0$ & & $1 \cdot 0$ & & $1 \cdot 0$ & \\
\hline $300-1800 \mathrm{ml} /$ week & 0.99 & $0.53,1.85$ & 0.79 & $0.39,1.63$ & 1.22 & $0.59,2.52$ & 1.80 & $0.90,3.56$ \\
\hline $300-600 \mathrm{ml} / \mathrm{d}$ & 0.77 & $0.51,1.14$ & 0.73 & $0.46,1.16$ & $0 \cdot 70$ & $0.42,1 \cdot 16$ & 0.92 & $0.54,1.57$ \\
\hline$>600 \mathrm{ml} / \mathrm{d}$ & 0.38 & $0.22,0.68$ & 0.42 & $0.22,0.79$ & 0.47 & $0.23,0.94$ & 1.25 & $0.68,2.29$ \\
\hline
\end{tabular}

H-TC, hypercholesterolaemia; H-TAG, hypertriacyclglycerolaemia; H-LDL-C, hyper-LDL-cholesterol; L-HDL-C, lower HDL-cholesterol.

${ }^{*}$ Adjusted for age; sex; education level; BMI; waist circumference; systolic blood pressure; diastolic blood pressure; levels of fasting plasma glucose, alanine aminotransferase, aspartate aminotransferase, creatinine and uric acid; physical activity; sitting time; smoking status; smoking pack-years; alcohol drinking status and weekly dietary intake of vegetables, fruit, soya food, milk, animal offal, lard and pickle.

$\uparrow 300 \mathrm{ml}$ equate to 1 pao.

$\ddagger$ Referent.

Table 5. Association of duration of tea consumption and amount of dried tea leaves brewed with dyslipidaemia* (Odds ratios and $95 \%$ confidence intervals)

\begin{tabular}{|c|c|c|c|c|c|c|c|c|}
\hline & \multicolumn{2}{|c|}{$\begin{array}{l}\text { Cases } \\
(n 1651)\end{array}$} & \multicolumn{2}{|c|}{$\begin{array}{l}\text { Controls } \\
(n \text { 1390) }\end{array}$} & \multirow[b]{2}{*}{ Crude OR } & \multirow[b]{2}{*}{$95 \% \mathrm{Cl}$} & \multirow[b]{2}{*}{ Adjusted OR $†$} & \multirow[b]{2}{*}{$95 \% \mathrm{Cl}$} \\
\hline & $n$ & $\%$ & $n$ & $\%$ & & & & \\
\hline Duration of tea consumption (years) & & & & & & & \multicolumn{2}{|c|}{$P_{\text {trend }}<0.001$} \\
\hline$<1 \ddagger$ & 147 & 8.9 & 78 & 5.6 & 1.0 & & 1.0 & \\
\hline $1-10$ & 362 & 21.9 & 401 & $28 \cdot 8$ & 0.48 & $0.35,0.65$ & 0.44 & $0.30,0.64$ \\
\hline $11-20$ & 502 & 30.4 & 490 & $35 \cdot 3$ & 0.54 & $0.40,0.74$ & 0.20 & $0.13,0.29$ \\
\hline$\geq 21$ & 640 & 38.8 & 421 & $30 \cdot 3$ & 0.81 & $0.60,1.09$ & 0.10 & $0.06,0.16$ \\
\hline Total tea leaves ( $\mathrm{g} / \mathrm{month})$ & & & & & & & \multicolumn{2}{|c|}{$P_{\text {trend }}<0.001$} \\
\hline$<1 \ddagger$ & 156 & $9 \cdot 4$ & 90 & 6.5 & $1 \cdot 0$ & & 1.0 & \\
\hline $1-750$ & 488 & $29 \cdot 6$ & 463 & $33 \cdot 3$ & 0.61 & $0.46,0.81$ & 0.35 & $0.25,0.49$ \\
\hline $751-1125$ & 497 & $30 \cdot 1$ & 458 & $32 \cdot 9$ & 0.63 & $0.47,0.84$ & 0.34 & $0.24,0.47$ \\
\hline$>1125$ & 510 & 30.9 & 379 & $27 \cdot 3$ & 0.78 & $0.58,1.04$ & 0.34 & $0.24,0.48$ \\
\hline Green tea leaves (g/month) & & & & & & & \multicolumn{2}{|c|}{$P_{\text {trend }}<0.001$} \\
\hline$<1 \ddagger$ & 1500 & 90.9 & 1201 & $86 \cdot 4$ & $1 \cdot 0$ & & 1.0 & \\
\hline $1-350$ & 36 & $2 \cdot 2$ & 48 & 3.5 & 0.60 & $0.39,0.93$ & 0.80 & $0.48,1.34$ \\
\hline $351-750$ & 59 & $3 \cdot 6$ & 57 & $4 \cdot 1$ & 0.83 & $0.57,1.20$ & 0.77 & $0.51,1.18$ \\
\hline$>750$ & 56 & 3.4 & 84 & $6 \cdot 0$ & 0.53 & $0.38,0.76$ & 0.37 & $0.25,0.54$ \\
\hline Oolong tea leaves ( $\mathrm{g} /$ month) & & & & & & & \multicolumn{2}{|c|}{$P_{\text {trend }}<0.001$} \\
\hline$<1 \ddagger$ & 225 & $13 \cdot 6$ & 166 & 11.9 & $1 \cdot 0$ & & 1.0 & \\
\hline $1-750$ & 488 & $29 \cdot 6$ & 462 & 33.2 & 0.78 & $0.62,0.99$ & 0.54 & $0.41,0.71$ \\
\hline $751-1125$ & 482 & 29.2 & 472 & 34.0 & 0.75 & $0.59,0.96$ & 0.47 & $0.35,0.61$ \\
\hline$>1125$ & 456 & $27 \cdot 6$ & 290 & 20.9 & $1 \cdot 16$ & $0.91,1.49$ & 0.60 & $0.45,0.81$ \\
\hline Black tea leaves (g/month) & & & & & & & \multicolumn{2}{|c|}{$P_{\text {trend }}=0.006$} \\
\hline$<1 \ddagger$ & 1509 & 91.4 & 1214 & $86 \cdot 7$ & $1 \cdot 0$ & & 1.0 & \\
\hline $1-300$ & 4 & 2.5 & 43 & 3.1 & 0.79 & $0.51,1.21$ & 1.09 & $0.67,1.78$ \\
\hline $301-600$ & 49 & $3 \cdot 0$ & 70 & $5 \cdot 0$ & 0.56 & $0.38,0.81$ & 0.55 & $0.36,0.82$ \\
\hline$>600$ & 51 & $3 \cdot 1$ & 63 & 4.5 & 0.65 & $0.45,0.95$ & 0.64 & $0.42,0.97$ \\
\hline
\end{tabular}

* The crude OR, adjusted OR and $P$ values refer to those of the controls.

†Adjusted for age; sex; education level; BMI; waist circumference; systolic blood pressure; diastolic blood pressure; levels of fasting plasma glucose, alanine aminotransferase, aspartate aminotransferase, creatinine and uric acid; physical activity; sitting time; smoking status; smoking pack-years; alcohol drinking status and weekly dietary intake of vegetables, fruit, soya food, milk, animal offal, lard and pickle.

$\ddagger$ Referent. 
Table 6. Adjusted association between duration of oolong tea consumption (in categories) and levels of blood lipids

\begin{tabular}{|c|c|c|c|c|}
\hline $\begin{array}{l}\text { Duration of } \\
\text { oolong tea } \\
\text { consumption } \\
\text { (years) }\end{array}$ & $n$ & $\begin{array}{l}\text { Percentage } \\
\text { of decrease in } \\
\text { blood levels* }\end{array}$ & $\begin{array}{c}\text { Absolute } \\
\text { decrease } \\
\text { in blood } \\
\text { levels } \\
(\mathrm{mmol} / \mathrm{l})\end{array}$ & $95 \% \mathrm{Cl} \dagger$ \\
\hline $\mathrm{TC}$ & & & \multicolumn{2}{|c|}{$P_{\text {trend }}=0.015$} \\
\hline$<1 \ddagger$ & 393 & & & \\
\hline $1-10$ & 691 & 0.40 & 0.02 & $-0.09,0.14$ \\
\hline $11-20$ & 940 & 1.01 & 0.05 & $-0.05,0.16$ \\
\hline$\geq 21$ & 1017 & 3.22 & 0.16 & $0.03,0.28$ \\
\hline $\mathrm{TAG}$ & & & \multicolumn{2}{|c|}{$P_{\text {trend }}=0.006$} \\
\hline$<1 \ddagger$ & 393 & & & \\
\hline $1-10$ & 691 & 0.63 & 0.01 & $-0.12,0.10$ \\
\hline $11-20$ & 940 & 1.89 & 0.03 & $-0.07,0.13$ \\
\hline$\geq 21$ & 1017 & 11.99 & 0.19 & $0.07,0.31$ \\
\hline LDL-C & & & \multicolumn{2}{|c|}{$P_{\text {trend }}<0.001$} \\
\hline$<1 \ddagger$ & 393 & & & \\
\hline $1-10$ & 691 & 1.06 & 0.03 & $-0.06,0.12$ \\
\hline $11-20$ & 940 & 3.87 & 0.11 & $0.02,0.19$ \\
\hline$\geq 21$ & 1017 & 6.69 & 0.19 & $0.09,0.30$ \\
\hline HDL-C & & & \multicolumn{2}{|c|}{$P_{\text {trend }}=0.655$} \\
\hline$<1 \ddagger$ & 393 & & & \\
\hline $1-10$ & 691 & -3.66 & -0.05 & $-0.10,0.01$ \\
\hline $11-20$ & 940 & -2.93 & -0.04 & $-0.08,0.01$ \\
\hline$\geq 21$ & 1017 & -0.73 & -0.01 & $-0.07,0.05$ \\
\hline
\end{tabular}

TC, total cholesterol; LDL-C, LDL-cholesterol; HDL-C, HDL-cholesterol.

* Calculated by the absolute reduction divided by the adjusted mean of the reference group.

†Data were adjusted for age; sex; education level; BMI; waist circumference; systolic blood pressure; diastolic blood pressure; levels of fasting plasma glucose, alanine aminotransferase, aspartate aminotransferase, creatinine and uric acid; physical activity; sitting time; smoking status; smoking pack-years; alcohol drinking status and weekly dietary intake of vegetables, fruit, soya food, milk, animal offal, lard and pickle.

$\ddagger$ Referent.

group, oolong tea consumption $\geq 21$ years was associated with a decrease in blood TC, TAG and LDL-C levels: $0.16 \mathrm{mmol} / \mathrm{l}$ (95\% CI 0.03, 0.28 mmol/1; 3.22\%); $0 \cdot 19 \mathrm{mmol} / 1$ (95\% CI 0.10, $0.39 \mathrm{mmol} / \mathrm{l} ; 11.99 \%) ; 0.19 \mathrm{mmol} / 1$ (95\% CI $0 \cdot 17,0.41 \mathrm{mmol} / \mathrm{l}$; 6.69\%), respectively, but not with HDL-C levels.

\section{Discussion}

The important role of dyslipidaemia in atherosclerosis and CHD has often been reported. High levels of plasma cholesterol and TAG lead to a high risk of CHD and atherosclerosis. This is the first study to document in detail the association between tea consumption and reduced dyslipidaemia risk in a population in southern China. Accurate measurements of long-term tea exposure involved the use of a validated and reliable questionnaire specifically developed for the southern Chinese population. In addition to recording the frequency of tea consumption, we obtained information on duration of tea drinking and mean quantity of dried tea leaves brewed. These exposure measures are important to determine the effect of habitual tea consumption. Moreover, the Shantou population consumes various types of tea, which allowed us to investigate their individual effects on dyslipidaemia risk, so the habitual tea consumption pattern would not be affected by the casecontrol status. We found that people who drank green, oolong and black tea daily had a significantly lower risk of dyslipidaemia and the risk was further reduced with longterm and increased-dose exposure to tea. Most importantly, the present results are robust and consistently demonstrate a dose-response relationship between duration of oolong tea consumption and blood TC, TAG and LDL-C levels but not HDL-C levels.

The protective effect of tea that we found is supported by experimental studies ${ }^{(8-10)}$. The effect is mainly due to the polyphenols present in tea. Tea polyphenols have various biological activities, including anti-inflammatory, anti-mutagenic, antioxidative and anti-tumour effects ${ }^{(28-31)}$. Recent studies ${ }^{(23,32,33)}$ in human subjects and animals have shown that tea consumption can lower plasma cholesterol and TAG levels. These studies ${ }^{(23,32,33)}$ have suggested that tea intake may protect against dyslipidaemia by suppressing fatty acid synthase. Tea polyphenols have been demonstrated in human breast carcinoma MCF-7 cells and hepatocellular carcinoma Hep-G2 cells $^{(32)}$.

Moreover, the order of the effect on the risk of dyslipidaemia for the three tea types is as follows: green tea $>$ oolong tea $>$ black tea. During fermentation, catechins are converted into the major black tea polyphenolic components theaflavins and thearubigins. The content of catechins, especially (-)-epigallocatechin, is higher in non-fermented green tea leaves than in partially fermented oolong tea leaves and fully fermented black tea leaves ${ }^{(34)}$. Animal studies have suggested that green tea catechins reduce lipid absorption in the intestines, promote faecal excretion of cholesterol and inhibit enzymes involved in hepatic cholesterol synthesis ${ }^{(35,36)}$.

Although green tea had the largest effect on the risk of dyslipidaemia, only oolong tea could reduce the levels of all lipids: H-TC, H-LDL, H-TAG and especially L-HDL. Besides catechins, oolong tea also contains high-molecular weight polyphenols such as OTPP produced during the process of semi-fermentation. It has been demonstrated that catechins can lower plasma TAG, TC and LDL-C levels but not HDL-C levels by inhibiting the activity of lipase ${ }^{(23,37)}$. That oolong tea increases HDL-C levels is inconsistent ${ }^{(23,37-39)}$. A previous animal study has found that oolong tea could increase HDL-C levels ${ }^{(39)}$, which may be related to the regulative action of oolong tea high-molecular weight polyphenols in lipoprotein metabolism. HDL can transport cholesterol from the surrounding tissues back to the liver for recycling or excretion in the form of bile acid and thus lower the levels of TC and LDL-C in the blood plasma. High levels of plasma cholesterol, LDL-C and VLDL-cholesterol are associated with a high risk of $\mathrm{CHD}$ and atherosclerosis ${ }^{(5,6)}$. CHD risk is positively associated with TC and LDL-C levels and inversely associated with HDL-C levels in middle-aged people ${ }^{(40,41)}$.

Shantou people mainly use locally grown tea leaves, which belong to the semi-fermented oolong tea series. Only this type of tea can offer the colour, fragrance and flavour required for Gongfu tea. The way Gongfu tea is prepared including the use of small tea sets probably contributes to the maintenance of the higher concentrations of polyphenols in Gongfu tea, which might explain why Gongfu tea has a greater lowering effect on lipids. Oolong tea varieties mostly used are Iron 
Buddha, narcissus and phoenix tea. Oolong tea has many health benefits in humans, such as reduction of CVD risk and antioxidant, anti-obesity and hypolipidaemic effects $^{(42-44)}$. This tea contains numerous kinds of polymerised polyphenols, the characteristic feature of oolong tea components, which are derived from tea catechins by the semi-fermentation process. The polymerised polyphenols present in oolong tea are not found in green tea and are different from those in black tea. In a previous in vivo study, OTPP have been found to significantly suppress both lymphatic TAG absorption and serum TAG elevation when a high-lipid diet is consumed ${ }^{(45)}$. The OTPP can also inhibit lipase activity by influencing intestinal absorption of dietary lipids, which is essential for lipid absorption, and faecal lipid excretion, without the side effects of polymerised polyphenols ${ }^{(45)}$. The inhibitory activity of OTPP on lipase is stronger than that of the total extracts derived from green tea in vitro, which could be explained by the ester-bound galloyl moieties ${ }^{(22)}$.

Dyslipidaemia is a complex systemic disorder, influenced by both lifestyle and dietary factors. Several randomised trials that did not control for diet did not reveal an effect of tea consumption on lipid levels ${ }^{(16,17)}$. Therefore, we investigated dietary and lifestyle factors to determine the effect of tea consumption on dyslipidaemia. Dietary assessment was based on subject self-reporting with a 125-item semi-quantitative FFQ developed for the southern Chinese population ${ }^{(25,26)}$. We found smoking, alcohol drinking status, sitting time, physical activity and dietary intake of vegetables, fruit, soya food, milk, animal offal, lard and pickle to be associated with dyslipidaemia. All these variables were accounted for and adjusted in the multivariate logistic regression analyses when analysing the relationship between tea consumption and dyslipidaemia. We also chose patients with newly diagnosed dyslipidaemia to control for recall bias and used face-to-face interviews to increase the response rate and improve the accuracy of answers. In addition, all the interviews were conducted by the same investigator to eliminate interviewer bias.

\section{Conclusions}

Oolong tea is the most widely consumed beverage in Shantou. We found that long-term oolong tea consumption can reduce the risk of H-TC, H-TAG and L-HDL in the Shantou population. Our data will be useful for planning effective education programmes to prevent CHD and other CVD. Nevertheless, further replications of the study are recommended before generalising the findings to other populations and countries.

The present study was supported by grants from Science and Technology Plans of Guangdong Province (2011B031800209), Medical Scientific Research Foundation of Guangdong Province (A2010388), the Joint Research Project of Clinical and Basic Courses, Shantou University Medical College (2010). The contents reflect only the authors' views. The administration of Science and Technology of Guangdong Province, Medical Scientific Research Foundation of Guangdong Province and Shantou University Medical College had no role in the design and analysis of the study or in the writing of this article.
The authors' contributions are as follows: D. Y. and Z. Z. conducted the survey, carried out the statistical analysis and drafted the manuscript; X. T. contributed to the conception and design of the study; Y. C., Y. L., X. L., S. L. and Y. C. coordinated the study and were involved in the questionnaire survey and anthropometric parameter measurement; Q. Z. contributed to the conception and design of the study and revised the manuscript. All authors read and approved the final manuscript.

None of the authors has any conflicts of interest to declare.

\section{References}

1. Murray CJ \& Lopez AD (1997) Mortality by cause for eight regions of the world: Global Burden of Disease Study. Lancet 349, 1269-1276.

2. Wu Z, Yao C, Zhao D, et al. (2001) Sino-MONICA project: a collaborative study on trends and determinants in cardiovascular diseases in China, Part i: morbidity and mortality monitoring. Circulation 103, 462-468.

3. Negishi H, Xu JW, Ikeda K, et al. (2004) Black and green tea polyphenols attenuate blood pressure increases in stroke-prone spontaneously hypertensive rats. J Nutr $\mathbf{1 3 4}$, $38-42$.

4. Yang TT \& Koo MW (1997) Hypocholesterolemic effects of Chinese tea. Pharmacol Res 35, 505-512.

5. Mukamal KJ, Maclure M, Muller JE, et al. (2002) Tea consumption and mortality after acute myocardial infarction. Circulation 105, 2476-2481.

6. Jain KS, Kathiravan MK, Somani RS, et al. (2007) The biology and chemistry of hyperlipidemia. Bioorg Med Chem 15, 4674-4699.

7. Peters U, Poole C \& Arab L (2001) Does tea affect cardiovascular disease? A meta-analysis. Am J Epidemiol 154, 495-503.

8. Akinyanju P \& Yudkin J (1967) Effect of coffee and tea on serum lipids in the rat. Nature 214, 426-427.

9. Chan PT, Fong WP, Cheung YL, et al. (1999) Jasmine green tea epicatechins are hypolipidemic in hamsters (Mesocricetus auratus) fed a high fat diet. J Nutr 129, 1094-1101.

10. Bursill CA \& Roach PD (2007) A green tea catechin extract upregulates the hepatic low-density lipoprotein receptor in rats. Lipids 42, 621-627.

11. Nagao T, Komine $Y$, Soga $S$, et al. (2005) Ingestion of a tea rich in catechins leads to a reduction in body fat and malondialdehyde-modified LDL in men. Am J Clin Nutr 81, 122-129.

12. Chan CC, Koo MW, Ng EH, et al. (2006) Effects of Chinese green tea on weight, and hormonal and biochemical profiles in obese patients with polycystic ovary syndrome a randomized placebo-controlled trial. J Soc Gynecol Investig 13, 63-68.

13. Nantz MP, Rowe CA, Bukowski JF, et al. (2009) Standardized capsule of Camellia sinensis lowers cardiovascular risk factors in a randomized, double-blind, placebo-controlled study. Nutrition 25, 147-154.

14. Nagao T, Meguro S, Hase T, et al. (2009) A catechin-rich beverage improves obesity and blood glucose control in patients with type 2 diabetes. Obesity 17, 310-317.

15. van het Hof KH, de Boer HS, Wiseman SA, et al. (1997) Consumption of green or black tea does not increase resistance of low-density lipoprotein to oxidation in humans. Am J Clin Nutr 66, 1125-1132. 
16. Bingham SA, Vorster H, Jerling JC, et al. (1997) Effect of black tea drinking on blood lipids, blood pressure and aspects of bowel habit. BrJ Nutr 78, 41-55.

17. McAnlis GT, McEneny J, Pearce J, et al. (1998) Black tea consumption does not protect low density lipoprotein from oxidative modification. Eur J Clin Nutr 52, 202-206.

18. Duffy SJ, Vita JA, Holbrook M, et al. (2001) Effect of acute and chronic tea consumption on platelet aggregation in patients with coronary artery disease. Arterioscler Thromb Vasc Biol 21, 1084-1089.

19. Frank J, George TW, Lodge JK, et al. (2009) Daily consumption of an aqueous green tea extract supplement does not impair liver function or alter cardiovascular disease risk biomarkers in healthy men. J Nutr 139, 58-62.

20. Stensvold I, Tverdal A, Solvoll K, et al. (1992) Tea consumption. Relationship to cholesterol, blood pressure, and coronary and total mortality. Prev Med 21, 546-553.

21. Kono S, Shinchi K, Ikeda N, et al. (1992) Green tea consumption and serum lipid profiles: a cross-sectional study in northern Kyushu, Japan. Prev Med 21, 526-531.

22. Nakai M, Fukui Y, Asami S, et al. (2005) Inhibitory effects of oolong tea polyphenols on pancreatic lipase in vitro. J Agric Food Chem 53, 4593-4598.

23. Kuo KL, Weng MS, Chiang CT, et al. (2005) Comparative studies on the hypolipidemic and growth suppressive effects of oolong, black, pu-erh, and green tea leaves in rats. J Agric Food Chem 53, 480-489.

24. Expert Panel on Detection, Evaluation, and Treatment of High Blood Cholesterol in Adults (2001) Executive Summary of the Third Report of the National Cholesterol Education Program (NCEP) Expert Panel on Detection, Evaluation, and Treatment of High Blood Cholesterol in Adults (Adult Treatment Panel III). JAMA 285, 2486-2497.

25. Ke L, Toshiro T, Fengyan S, et al. (2005) Relative validity of a semi-quantitative food frequency questionnaire versus 3 day weighed diet records in middle-aged inhabitants in Chaoshan area, China. Asian Pac J Cancer Prev 6, 376-381.

26. Song FY, Toshiro T, Li K, et al. (2005) Development of a semi-quantitative food frequency questionnaire for middle-aged inhabitants in the Chaoshan area, China. World J Gastroenterol 11, 4078-4084.

27. Jian L, Xie LP, Lee AH, et al. (2004) Protective effect of green tea against prostate cancer: a case-control study in southeast China. Int J Cancer 108, 130-135.

28. Wiseman SA, Balentine DA \& Frei B (1997) Antioxidants in tea. Crit Rev Food Sci Nutr 37, 705-718.

29. Mukhtar H \& Ahmad N (2000) Tea polyphenols: prevention of cancer and optimizing health. Am J Clin Nutr 71, 1698S-1702S, (discussion 1703S-1704S).
30. Balentine DA, Wiseman SA \& Bouwens LC (1997) The chemistry of tea flavonoids. Crit Rev Food Sci Nutr 37, 693-704.

31. Kurahashi N, Sasazuki S, Iwasaki M, et al. (2008) Green tea consumption and prostate cancer risk in Japanese men: a prospective study. Am J Epidemiol 167, 71-77.

32. Yeh CW, Chen WJ, Chiang CT, et al. (2003) Suppression of fatty acid synthase in MCF-7 breast cancer cells by tea and tea polyphenols: a possible mechanism for their hypolipidemic effects. Pharmacogenomics J 3, 267-276.

33. Huang HC \& Lin JK (2012) Pu-erh tea, green tea, and black tea suppresses hyperlipidemia, hyperleptinemia and fatty acid synthase through activating AMPK in rats fed a high-fructose diet. Food Funct 3, 170-177.

34. Cabrera C, Gimenez R \& Lopez MC (2003) Determination of tea components with antioxidant activity. J Agric Food Chem 51, $4427-4435$.

35. Ikeda I, Imasato Y, Sasaki E, et al. (1992) Tea catechins decrease micellar solubility and intestinal absorption of cholesterol in rats. Biochim Biophys Acta 1127, 141-146.

36. Bursill CA, Abbey M \& Roach PD (2007) A green tea extract lowers plasma cholesterol by inhibiting cholesterol synthesis and upregulating the LDL receptor in the cholesterol-fed rabbit. Atherosclerosis 193, 86-93.

37. Yang M, Wang C \& Chen H (2001) Green, oolong and black tea extracts modulate lipid metabolism in hyperlipidemia rats fed high-sucrose diet. J Nutr Biochem 12, 14-20.

38. He RR, Chen L, Lin BH, et al. (2009) Beneficial effects of oolong tea consumption on diet-induced overweight and obese subjects. Chin J Integr Med 15, 34-41.

39. Matsuda H, Chisaka T, Kubomura Y, et al. (1986) Effects of crude drugs on experimental hypercholesterolemia. I. Tea and its active principles. J Ethnopharmacol 17, 213-224.

40. Boshuizen HC, Lanti M, Menotti A, et al. (2007) Effects of past and recent blood pressure and cholesterol level on coronary heart disease and stroke mortality, accounting for measurement error. Am J Epidemiol 165, 398-409.

41. Iso H, Naito Y, Sato S, et al. (2001) Serum triglycerides and risk of coronary heart disease among Japanese men and women. Am J Epidemiol 153, 490-499.

42. Han LK, Takaku T, Li J, et al. (1999) Anti-obesity action of oolong tea. Int J Obes Relat Metab Disord 23, 98-105.

43. Kurihara H, Fukami H, Toyoda $\mathrm{Y}$, et al. (2003) Inhibitory effect of oolong tea on the oxidative state of low density lipoprotein (LDL). Biol Pharm Bull 26, 739-742.

44. Kuroda Y \& Hara Y (1999) Antimutagenic and anticarcinogenic activity of tea polyphenols. Mutat Res 436, 69-97.

45. Toyoda-Ono Y, Yoshimura M, Nakai M, et al. (2007) Suppression of postprandial hypertriglyceridemia in rats and mice by oolong tea polymerized polyphenols. Biosci Biotechnol Biochem 71, 971-976. 